\title{
Análise da força muscular em idosas praticantes e não praticantes de atividade física sistematizada
}

\section{Analysis of muscular strength in the elderly practitioners and non-practitioners of systematized physical activity}

\author{
Anne Jacqueline Souza Santos ${ }^{1}$ \\ Claudiney Silva Ferreira ${ }^{2}$ \\ Amanda Mota Lacerda ${ }^{3}$ \\ João Lucas Rodrigues dos Santos ${ }^{4}$ \\ Mariana Rocha Alves 5 \\ Magda Mendes Vieira ${ }^{6}$ \\ Vinicius Dias Rodrigues ${ }^{7}$
}

Resumo: Introdução: A prática regular de atividade física é fundamental para idosos. A diminuição da força e da potência do músculo pode influenciar na diminuição de autonomia, bem-estar e da qualidade de vida dos mesmos. A perda da força e da massa muscular predispõe os idosos a uma limitação funcional, fator predisponente para muitos processos patológicos associados ao aumento da morbidade e mortalidade. Objetivo: Analisar a força muscular em idosos praticantes e não praticantes de atividades físicas sistematizadas. Metodologia: Trata-se

\footnotetext{
${ }^{1}$ Educadora física - Funorte. Minas Gerais - Brasil. $\triangle$ aninhajacky@yahoo.com.br . (D) https://orcid.org/00000003-0476-9661

${ }^{2}$ Educador físico - Funorte. Minas Gerais - Brasil. $\bowtie$ claudineypim@yahoo.com.br . (D) https://orcid.org/00000001-6446-884X

${ }_{3}^{3}$ Mestre em ciências da saúde (Unimontes). Minas Gerais - Brasil. $₫$ amandamlacerda@yahoo.com.br https://orcid.org/0000-0001-5333-4686

${ }^{4}$ Mestre em ciências da saúde (Unimontes). Minas Gerais - Brasil. $\square$ joaofadetec@gmail.com https://orcid.org/0000-0002-1724-3784

5 Mestre em medicina (UFF). Professora da Faculdade verde norte. Minas Gerais - Brasil. $\square$ marianarochaalves13@gmail.com (D) https://orcid.org/0000-0002-2673-3160

${ }^{6}$ Doutora em ciências da saúde (Unimontes). Minas Gerais - Brasil. $\square$ magdamendesvieira@hotmail.com https://orcid.org/0000-0001-5650-9787

${ }^{7}$ Doutor em ciências da saúde (Unimontes). Professor da Universidade Estadual de Montes Claros e Faculdades unidas do Norte de Minas Gerais. Minas Gerais - Brasil. $₫$ viniciuslabex@hotmail.com . https://orcid.org/0000-0002-6959-5992
}

\begin{tabular}{c|ccc} 
Recebido em & Aceito em & Correções em & Publicado em \\
$28 / 11 / 2018$ & $02 / 11 / 2019$ & $21 / 09 / 2020$ & $14 / 12 / 2020$
\end{tabular}


de uma pesquisa descritiva, quantitativa e de corte transversal. Este estudo foi realizado na cidade de Montes Claros - MG, com população idosa na faixa etária de 65 a 75 anos de idade. Os indivíduos foram divididos em dois grupos, sendo inclusos no G1 idosas praticantes de atividades físicas regulares, e no G2 idosas não praticantes de atividades físicas. Todos os dados foram coletados a partir da investigação das variáveis, digitalizados e analisados no programa estatístico (SPSS $\left.{ }^{\circledR}, 20.0\right)$. O presente estudo obteve aprovação do Comitê de Ética em Pesquisa das Faculdades Integradas do Norte de Minas. Nesta pesquisa, foram realizados testes de força muscular de preensão manual, e a comparação realizada a partir da média relativa, média absoluta, máxima relativa e máxima absoluta para ambas as mãos (direita e esquerda). Resultados: Idosas ativas tinham maior força de pressão manual, significativa quando comparadas com as idosas sedentárias. Conclusão: Verificou-se que o exercício físico regular pode ter contribuído para manutenção ou aumento da força muscular no grupo de idosas ativas. Palavras-chave: Força Muscular. Idosos. Sarcopenia. Atividade Física.

Abstract: Introduction: Regular physical activity is essential for the elderly. The decrease in muscle strength and power can influence the decrease in autonomy, well-being and quality of life. The loss of strength and muscle mass predisposes the elderly to a functional limitation, a predisposing factor for many pathological processes associated with increased morbidity and mortality. Objective: To analyze muscle strength in elderly practicing and non-practicing physical activity. Methodology: This is a descriptive, quantitative and cross-sectional research. This study was performed in the city of Montes Claros - MG, with an elderly population aged from 65 to 75 years old. The individuals were divided into two groups, being included in G1 elderly women who practice regular physical activities, and in G2 elderly women who do not practice physical activities. All data were collected from the investigation of the variables, digitized and analyzed using the statistical program (SPSS $®$, 20.0). This study was approved by the Research Ethics Committee of the Integrated Colleges of Northern Minas Gerais. In this research, handgrip muscle strength tests were performed, and the comparison was made from the relative mean, absolute mean, relative maximum and absolute maximum for both hands (right and left). Results: The results show that the active elderly have a bigger manual pressure force when bought with the sedentary elderly. Conclusion: We can understand that possibly regular physical exercise may have contributed to maintenance or increase of muscle strength in the group of active elderly women. 
Keywords: Muscular Strength, Elderly, Sarcopenia, Physical Activity

\section{INTRODUÇÃO}

No Brasil, de acordo com a Lei $\mathrm{n}^{\circ}$ 8.842, compreende-se por indivíduo idoso aquele que tem a idade igual ou superior a 60 anos. O crescimento dessa população acontece de forma acelerada e pode ser considerado um fenômeno de abrangência mundia ${ }^{1}$. No ano de 1950 , o Brasil aparecia como o $16^{\circ}$ país em quantidade populacional acima dos 60 anos de idade, contudo, a projeção é de que o país suba para a $6^{\circ}$ posição no ranking mundial no ano de 2025. A cada ano, uma média de 650 mil brasileiros entra para a população idosa no país e, grande parte, possui doenças crônicas, alguns, inclusive, com limitações funcionais ${ }^{2 ; 3}$.

O envelhecimento tem sido descrito como um processo, ou conjunto de processos, inerente a todos os seres vivos e que se expressa pela perda da capacidade de adaptação e pela diminuição da funcionalidade ${ }^{4}$. O processo de envelhecimento fisiológico promove uma diminuição das reservas funcionais do indivíduo ${ }^{5}$, acarretando mudanças morfofisiológicas, funcionais e bioquímicas, tornando-o mais suscetível a agressões intrínsecas e extrínsecas ${ }^{6}$. Entre as principais alterações observadas, está o decréscimo da função muscular, que afeta diretamente a capacidade de realizar tarefas do dia a dia, diminuindo a independência funcional e refletindo negativamente na qualidade de vida do idoso ${ }^{7}$.

Com o aumento da população idosa e o aumento da expectativa de vida, começam a surgir com maior frequência doenças e comorbidades relacionadas ao processo de envelhecimento, como a sarcopenia ${ }^{8}$. O termo sarcopenia (em grego, sark = carne;penia $=$ perda) foi pioneiramente utilizado por Irwin H. Rosenberg, para se referir à perda de massa muscular relacionada à idade, associada à perda de função $0^{8 ; 9}$.

Para Larsson, Grimby e Karlsson ${ }^{10}$, o maior índice de força muscular é alcançado por volta dos 30 anos, o qual se mantém estável até a quinta década, havendo redução na força muscular em torno de $15 \%$ entre a quinta e a sétima década, com acentuação ainda maior (30\%) após os 80 anos de idade. A redução de massa muscular (sarcopenia) pode gerar limitações funcionais (dinapenia) que acarretam perda da independência, quedas e fraturas ${ }^{11 ; 12}$. As consequências negativas da sarcopenia e dinapenia na população idosa se expressam nos elevados índices de morbimortalidade ${ }^{13 ;}$, no aumento do número de assistências sociais e sanitárias ${ }^{14}$ e nos registros de incapacidade física de idoso ${ }^{15 ; 12 ; 16}$. 
A incapacidade funcional exerce grande efeito negativo no bem-estar individual, gerando mais necessidade de assistência à saúde e cuidados por longos períodos ${ }^{18}$. Incapacidade funcional é comumente definida como a restrição da capacidade do indivíduo de desempenhar atividades normais da vida diária. Refere-se também a limitações específicas no desempenho de papéis socialmente definidos e de tarefas dentro de um ambiente sociocultural e físico particular. Estão incluídas as atividades básicas e instrumentais de vida diária, os papéis no trabalho, nas atividades não-ocupacionais, nos recreativos ou de lazer ${ }^{17}$;

A atrofia muscular em indivíduos idosos pode ser amenizada com a prática regular de exercício físico. Homens e mulheres nessa faixa etária, com menor atividade física apresentam também menor massa muscular e maior prevalência de incapacidade física ${ }^{11}$.Estudos demonstraram que idosos sarcopênicos que se submeteram a treinamento de resistência melhoraram seu desempenho nas atividades funcionais, podendo atenuar ou mesmo reverter a diminuição de massa e força muscular ${ }^{19 ; 20 ; 21}$.

Um instrumento muito utilizado para se estimar a força muscular global em idosos é à força de preensão palmar. De forma geral, os idosos que apresentam força de preensão manual reduzida são sedentários, possuem déficits de massa corporal, apresentam problemas de saúde e limitações funcionais em atividades que exigem a participação dos membros superiores e inferiores. Portanto, essa medida deixa de ser apenas uma simples medida da força da mão, limitada à avaliação do membro superior e passa a apresentar outras implicações clínicas ${ }^{21 ; 22}$. $\mathrm{O}$ aumento na expectativa de vida e a vulnerabilidade a doenças crônico-degenerativas e comorbidades remetem à busca de intervenções que minimizem os efeitos deletérios do envelhecimento, com consequente melhoria da qualidade de vida ${ }^{23}$.

Assim, o presente estudo objetivou verificar a diferença da força de preensão manual em idosas praticantes e não praticantes de atividade física sistematizada.

\section{METODOLOGIA}

Trata-se de um estudo descritivo, com abordagem quantitativa de corte transversal na cidade de Montes Claros - MG, com uma amostra composta por idosos do sexo feminino, que se encontravam na faixa etária de 65 a 75 anos de idade. A amostra foi selecionada por conveniência (disponibilidade de participação), totalizando 85 indivíduos, que foram divididos em dois grupos, praticantes e não praticantes/ativos e sedentários. 
O primeiro grupo (G1), compôs-se por idosas ativas, praticantes de atividade física regular a mais de seis meses e o segundo grupo $(\mathrm{G} 2)$ composto por idosas ativas não praticantes de atividade física regular. Foram excluídas do estudo idosas que não assinaram o Termo de consentimento Livre e Esclarecido e/ou faltaram no dia da coleta. A amostra final foi composta por 85 indivíduos.

Para a coleta de dados a participante avaliada foi posicionada em pé, no centro da plataforma da balança, de costas para a escala da mesma (balança mecânica antropométrica Micheletti), com postura ereta e com o olhar em um ponto fixo à sua frente.

A medição da estatura foi realizada com o estadiômetro acoplado à balança. Para tanto, a participante, descalço, foi posicionada em posição ortostática (em pé), pés unidos, no centro da plataforma, braços ao longo do corpo e a cabeça orientada no plano de Frankfurt paralela ao solo, realizando uma inspiração máxima, permanecendo em apnéia por 3 segundos no momento do registro da estatura corporal ${ }^{23 ; 24}$.

Realizou-se o teste de força de preensão manual com o dinamômetro Manual50Kgf (Crown manual-50kgf). Para a medição da força de preensão manual, a participante foi posicionada sentada de forma confortável, posicionado com o ombro levemente aduzido; cotovelo fletido a $90^{\circ}$; antebraço em posição neutra e com a posição dos punhos variando de $0^{\circ}$ a $30^{\circ}$. A idosa avaliada apertou o dinamômetro com sua força máxima. O método utilizado para cálculo dos valores foi à média em triplicata para cada avaliada participante, respeitando o número de medidas e o tempo de descanso entre as tentativas de aproximadamente 30 segundos ${ }^{23}$.

Após a coleta, os dados foram analisados pelo programa estatístico SPSS ${ }^{\circledR}$, versão 20.0 para Windows e, inicialmente, foram empregados métodos de estatística descritiva (média e desvio padrão). Para verificar a normalidade das amostras, realizou-se o teste de KolmogorovSmirnov. Após a verificação, com a comprovação de que os dados seguiam distribuição normal, o teste t de student pareado e análise de confiabilidade foram aplicados, considerando o nível de confiança de $95 \%(\mathrm{p}<0,05)$.

Por tratar-se de uma pesquisa envolvendo seres humanos, este estudo atendeu às normas estabelecidas pela Resolução 466/12. O projeto de pesquisa foi submetido ao Comitê de Ética de Pesquisa da Faculdades Integradas do Norte de Minas (FUNORTE), e obteve parecer favorável $\mathrm{n}^{\mathrm{o}}$ 1.897.677. Todas as idosas envolvidas neste estudo assinaram o Termo de Consentimento Livre e Esclarecido, após receber esclarecimentos relativos as características da pesquisa, o sigilo dos dados obtidos e a sua livre decisão de participação no presente estudo. 


\section{RESULTADOS}

Os resultados obtidos na Tabela 1 abordam os dados de caracterização das participantes, não apresentando diferença estatisticamente significativa em relação à idade, peso corporal, estatura e índice de massa corporal. Participaram deste estudo 85 idosos do gênero feminino; com média de idade entre $68,6286 \pm 2,95$ para idosas ativas e $69,58 \pm 3,15$ para as sedentárias. No aspecto da caracterização do peso corporal $(\mathrm{kg})$ as idosas ativas apresentaram média de

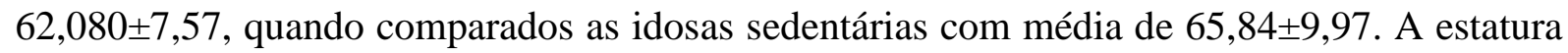

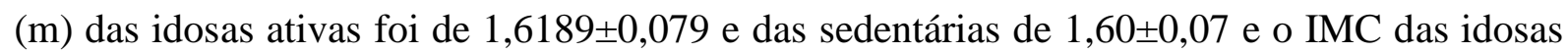

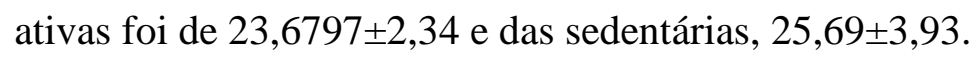

Tabela 1 - Caracterização da idade (anos), peso corporal (kg), estatura (metros) e índice de massa corporal $\left(\mathrm{kg} / \mathrm{m}^{2}\right)$ dos indivíduos ativos e sedentários.

\begin{tabular}{lccc}
\hline & $\begin{array}{c}\text { Ativos } \\
(\mathrm{n}=35)\end{array}$ & $\begin{array}{c}\text { Sedentários } \\
(\mathrm{n}=50)\end{array}$ & \\
\hline & $\begin{array}{c}\text { Média } \pm \text { Desvio } \\
\text { padrão }\end{array}$ & $\begin{array}{c}\text { Média } \pm \text { Desvio } \\
\text { padrão }\end{array}$ & Significância \\
\hline Idade (anos) & $68,6286 \pm 2,95$ & $69,58 \pm 3,15$ & $\mathrm{p}=0,890$ \\
Peso $(\mathrm{kg})$ & $62,0800 \pm 7,57$ & $65,84 \pm 9,97$ & $\mathrm{p}=0,640$ \\
Estatura $(\mathrm{m})$ & $1,6189 \pm 0,079$ & $1,60 \pm 0,07$ & $\mathrm{p}=0,870$ \\
$\mathrm{IMC}\left(\mathrm{kg} / \mathrm{m}^{2}\right)$ & $23,6797 \pm 2,34$ & $25,69 \pm 3,93$ & $\mathrm{p}=0,490$ \\
\hline
\end{tabular}

Os resultados apresentados na Tabela 2 foram coletados pelo dinamômetro manual e apresentam confiabilidade para mensuração da força muscular de pressão. $O$ teste de preensão manual realizado nos grupos de idosas ativas e sedentárias foi confiável, pois os valores obtidos nas análises inferenciais foram de correlações intraclasse entre os membros direito e esquerdo de 0,909 e 0,912 respectivamente.

Tabela 2 - Resultado dos cálculos do coeficiente de correlação intraclasse para mensuração da força muscular de preensão manual.

$\mathrm{n}$

85
Quantidade de teste-reteste

3
Correlação intraclasse

0.909 p

0.000 * 
Foram realizados os testes de força muscular de preensão manual, e a comparação foi realizada a partir da média relativa, média absoluta, máxima relativa e máxima absoluta para ambas as mãos (direita e esquerda) dos grupos de idosas ativas e sedentárias, no qual o resultado mostrou que as idosas ativas possuíam maior força de pressão manual significativamente (Tabela 3).

Tabela 3 - Comparação da força muscular de pressão manual em os indivíduos ativos e sedentários.

\begin{tabular}{lccc}
\hline & Ativos $(\mathrm{n}=35)$ & Sedentários $(\mathrm{n}=50)$ & \\
\hline \multicolumn{1}{c}{ Variáveis } & $\begin{array}{c}\text { Média } \\
\pm \text { Desvio } \\
\text { padrão }\end{array}$ & $\begin{array}{c}\text { Média } \\
\pm \text { Desvio padrão }\end{array}$ & Significância \\
& $0,39 \pm 0,07$ & $0,20 \pm 0,50$ & $\mathrm{p}=0,000$ \\
\hline FMPMMRD & $0,38 \pm 0,6$ & $0,19 \pm 0,4$ & $\mathrm{p}=0,000$ \\
FMPMMRE & $24,58 \pm 4,10$ & $12,95 \pm 3,12$ & $\mathrm{p}=0,000$ \\
FMPMMAD & $23,98 \pm 41,17$ & $12,46 \pm 2,93$ & $\mathrm{p}=0,000$ \\
FMPMMAE & $0,41 \pm 0,74$ & $0,21 \pm 0,06$ & $\mathrm{p}=0,000$ \\
FMPMMRD2 & $0,40 \pm 0,06$ & $0,21 \pm 0,05$ & $\mathrm{p}=0,000$ \\
FMPMMRE2 & $25,45 \pm 4,18$ & $14,25 \pm 3,16$ & $\mathrm{p}=0,000$ \\
FMPMMAD2 & $25,02 \pm 4,03$ & $13,61 \pm 3,03$ & $\mathrm{p}=0,000$ \\
FMPMMAE2 &
\end{tabular}

Média e desvio padrão. Foi utilizado o teste t de student independente. Força muscular de pressão manual média relativa direito $(\mathrm{Kgf})=$ FMPMMRD, Força muscular de pressão manual média relativa esquerda $(\mathrm{Kgf})=$ FMPMMRE, Força muscular de pressão manual média absoluta direito $(\mathrm{Kgf})=$ FMPMMAD, Força muscular de pressão manual média absoluta esquerdo $(\mathrm{Kgf})$ = FMPMMAE,Força muscular de pressão manual máxima relativa direito $(\mathrm{Kgf})=$ FMPMMRD2, Força muscular de pressão manual máxima relativa esquerda $(\mathrm{Kgf})=$ FMPMMRE2, Força muscular de pressão manual máxima absoluta direito $(\mathrm{Kgf})=$ FMPMMAD2, Força muscular de pressão manual máxima absoluta esquerdo $(\mathrm{Kgf})=$ FMPMMAE2.

\section{DISCUSSÃO}

A homogeneidade de uma amostra ou universo amostral quando inserido em uma pesquisa é algo que deve ser levado em consideração durante a construção e desempenho de 
um trabalho como um todo, levando em consideração que amostras homogêneas, a depender das avaliações pretendidas, faz com que o trabalho possua uma maior força de comparação e resultados mais confiáveis ${ }^{25 ; 26}$.

A seleção amostral apresentada no presente estudo deu-se levando em consideração, além da disponibilidade em participar do estudo, via seleção de mulheres idosas que se encontrassem em uma faixa etária similar, garantindo assim com que o valor de p destacado na tabela 1 apresente-se como não significativos.

De acordo com a literatura, o processo de envelhecimento, por si, pode acarretar o declínio da aptidão física, que se agrava com o sedentarismo, tornando os idosos dependentes de cuidados de outros, já que a inatividade física é um dos fatores que contribui para a redução da aptidão funcional e sarcopenia ${ }^{24}$.

Estudos têm mostrado que homens e mulheres ativos conseguem manter a força muscularem com níveis maiores que os inativos, reforçando que a atividade física está diretamente relacionada à força muscular ${ }^{8 ; 27}$

O sedentarismo pode contribuir para a perda funcional, propiciada pelo próprio déficit de massa muscular e força, que se dão naturalmente com o passar do tempo, diminuindo a aptidão e desempenho físico, o que os torna ainda mais inativos, dificultando atividades cotidianas e diminuindo a qualidade de $\operatorname{vida}^{28 ; 29}$.

Observações realizadas durante este trabalho revelaram que indivíduos sedentários têm sua força de preensão manual menor que a força de preensão observada nos indivíduos praticantes de atividade física, sendo todos os valores de $\mathrm{p}$ significativos.

Segundo Lenardt, et al. ${ }^{30}$ a redução da força de preensão manual tem sido explorada como uma manifestação inicial de fragilidade e pode estar presente antes mesmo do surgimento de outras incapacidades funcionais, sua investigação com amostra probabilística de idosos comunitários com 65 anos e mais, integrantes de um estudo populacional sobre fragilidade, identificou que os idosos sedentários, ou com baixo gasto calórico semanal, e os idosos mais velhos diferenciaram-se dos idosos ativos e dos mais jovens quanto à fadiga, à força de preensão e à velocidade da marcha ${ }^{30}$.

O uso do dinamômetro manual para acessar a força de preensão manual é tido como um dos métodos de menor complexidade quanto à utilização e maior eficácia para permitir avaliação da força física e seu declínio em indivíduos idosos, sendo tal técnica utilizada amplamente em diversos estudos ${ }^{31 ; 32}$, sendo este o motivo de escolha da técnica pela nossa equipe. 
Focar em um "envelhecimento ativo" faz-se cada vez mais importante, pois, a atividade durante o envelhecimento possui impacto sobre a autonomia funcional de idosos, sendo a perda desta autonomia responsável por características como diminuição na necessidade de cuidados, incapacidade de realização de atividades cotidianas e ainda distúrbios de ordem mental/comportamental, como ansiedade e depressão, impactando assim, diretamente, na qualidade de vida ${ }^{8 ; 17}$.

A redução progressiva da massa muscular em idosos tem relação com a incapacidade física, assim, é recomendado que os profissionais de saúde identifiquem alterações funcionais e musculares nos idosos, em especial nos longevos, pois esses podem predizer os indivíduos frágeis e pré-frágeis ${ }^{33 ;} 34$.

Considerando as características fisiológicas e os dados encontrados no presente estudo, é possível afirmar que o programa regular de exercício físico contribui para a manutenção da força de preensão de ambas as mãos, evitando os prejuízos decorrentes da perda fisiológica natural da idade.

Para um idoso realizar suas tarefas cotidianas como subir escadas, carregar suas compras e abaixar-se, há a necessidade de pouca aptidão cardiovascular, e muito mais de um conjunto de capacidades como força muscular, resistência muscular localizada e flexibilidade, conjunto este denominado de "aptidão muscular" 8; 30 .

Neste estudo, pode-se observar aumento da força muscular entre mulheres praticantes de atividade física, e esse aumento é um efeito que pode atuar como paliativo ou reversor da perda acentuada de aptidão física e posterior incapacidade funcional/sarcopenia.

O treinamento de força é uma alternativa eficiente que pode minimizar ou retardar o processo de sarcopenia por obter significantes respostas neuromusculares (hipertrofia muscular e força muscular), por meio do aumento da capacidade contrátil dos músculos esqueléticos ${ }^{35}$; 36 .

\section{CONCLUSÃO}

Os resultados do estudo apresentado mostraram que, idosos ativos possuem maior força muscular manual quando comparados aos idosos sedentários, sendo esta uma boa fonte prognóstica de ausência de quadro sarcopênico. Assim, podemos entender que a atividade física sistematizada praticada por indivíduos idosos contribui para manutenção ou aumento da força muscular quando comparados indivíduos ativos com indivíduos sedentários. 
O presente estudo apresenta também algumas limitações, estando entre elas o uso da seleção amostral por conveniência e o pequeno número de indivíduos selecionados para a amostragem. É também uma limitação a utilização única e exclusiva do dinamômetro manual como parâmetro avaliativo, podendo outras medidas antropométricas serem associadas às avaliações.

Para esta pesquisa, não houve financiado, não sendo resultado de dissertação de mestrado ou tese de doutorado e não houve conflitos de interesse.

\section{REFERÊNCIAS}

1 KARSCH, U. M. Idosos dependentes: famílias e cuidadores. Cadernos de Saúde Pública, v. 19, p. 861-866, 2003. ISSN 0102-311X. Disponível em: < http://www.scielo.br/scielo.php?script=sci_arttext\&pid=S0102$\underline{311 X 2003000300019 \& \text { nrm }=\text { iso }}>$.

2 BRASIL. Lei $\mathrm{n}^{\circ}$ 8.842, de 4 de janeiro de 1994. Dispõe sobre a Política Nacional do Idoso, cria o Conselho Nacional do Idoso e dá outras providências. Diário oficial da União, 1994.

3 RP, V. Envelhecimento populacional e as informações de saúde do PNAD: demandas e desafios contemporâneos. Cad Saúde Pública, v. 23, n. 1, p. 2463-6, 2007.

4 CRISTOPOLISKI, F. et al. Stretching exercise program improves gait in the elderly. Gerontology, v. 55, n. 6, p. 614-620, 2009. ISSN 0304-324X.

5 BARBOSA, S. M. Estudo do equilíbrio em idosos através da fotogrametria computadorizada. Fisioterapia Brasil, v. 2, n. 3, p. 178-182, 2016 . ISSN 2526-9747.

6 LACOURT, M. X.; MARINI, L. L. Decréscimo da função muscular decorrente do envelhecimento e a influência na qualidade de vida do idoso: uma revisão de literatura. Revista Brasileira de Ciências do Envelhecimento Humano, v. 3, n. 1, 2006. ISSN 2317-6695.

7 SILVA, T. A. D. A. et al. Sarcopenia associada ao envelhecimento: aspectos etiológicos e opções terapêuticas. Revista Brasileira de Reumatologia, v. 46, n. 6, p. 391-397, 2006. ISSN 0482-5004.

FERREIRA, O. G. L. et al. Envelhecimento ativo e sua relação com a independência funcional. Texto \& Contexto-Enfermagem, v. 21, n. 3, p. 513-518, 2012. ISSN 01040707.

9 ROSENBERG, I. H. Sarcopenia: origins and clinical relevance. The Journal of nutrition, v. 127, n. 5, p. 990S-991S, 1997. ISSN 0022-3166. 
LARSSON, L.; GRIMBY, G.; KARLSSON, J. Muscle strength and speed of movement in relation to age and muscle morphology. Journal of Applied Physiology, v. 46, n. 3, p. 451-456, 1979. ISSN 8750-7587.

JANSSEN, I.; HEYMSFIELD, S. B.; ROSS, R. Low relative skeletal muscle mass (sarcopenia) in older persons is associated with functional impairment and physical disability. Journal of the American Geriatrics Society, v. 50, n. 5, p. 889-896, 2002. ISSN 0002-8614.

SAYER, A. A. et al. Type 2 diabetes, muscle strength, and impaired physical function: the tip of the iceberg? Diabetes care, v. 28, n. 10, p. 2541-2542, 2005. ISSN 01495992.

GALE, C. R. et al. Grip strength, body composition, and mortality. International journal of epidemiology, v. 36, n. 1, p. 228-235, 2007. ISSN 1464-3685.

JANSSEN, I. et al. The healthcare costs of sarcopenia in the United States. Journal of the American Geriatrics Society, v. 52, n. 1, p. 80-85, 2004. ISSN 0002-8614.

GOODPASTER, B. H. et al. The loss of skeletal muscle strength, mass, and quality in older adults: the health, aging and body composition study. The Journals of Gerontology Series A: Biological Sciences and Medical Sciences, v. 61, n. 10, p. 1059-1064, 2006. ISSN 1758-535X.

DELMONICO, M. J. et al. Alternative definitions of sarcopenia, lower extremity performance, and functional impairment with aging in older men and women. Journal of the American Geriatrics Society, v. 55, n. 5, p. 769-774, 2007. ISSN 0002-8614.

MARIANO, E. R. et al. Força muscular e qualidade de vida em idosas. Revista Brasileira de geriatria e gerontologia, v. 16, n. 4, p. 805-811, 2013. ISSN 1809-9823.

RABELO, D. F.; CARDOSO, C. M. Auto-eficácia, doenças crônicas e incapacidade funcional na velhice. Psico-USF, v. 12, n. 1, p. 75-81, 2007. ISSN 1413-8271.

ROUBENOFF, R. Origins and clinical relevance of sarcopenia. Canadian journal of applied physiology, v. 26, n. 1, p. 78-89, 2001. ISSN 1066-7814.

FRISARD, M. I. et al. Physical activity level and physical functionality in nonagenarians compared to individuals aged 60-74 years. The Journals of Gerontology Series A: Biological Sciences and Medical Sciences, v. 62, n. 7, p. 783788, 2007. ISSN 1758-535X.

BRIEF, A. Envelhecimento, força muscular e atividade física: uma breve revisão bibliográfica. Revista Científica FacMais, v. 2, n. 1, 2012.

EVANS, W. J. Effects of exercise on senescent muscle. Clinical Orthopaedics and Related Research®, v. 403, p. S211-S220, 2002. ISSN 0009-921X. 
SHECHTMAN, O. et al. Grip strength in the frail elderly. American journal of physical medicine \& rehabilitation, v. 83, n. 11, p. 819-826, 2004. ISSN 0894-9115.

MARINS, J. C. B.; GIANNICHI, R. S. Avaliação e prescrição de atividade física: guia prático. Shape, 1998. ISBN 8585253126.

NOGUEIRA, D. A.; PEREIRA, G. M. Desempenho de testes para homogeneidade de variâncias em delineamentos inteiramente casualizados. Sigmae, v. 2, n. 1, p. 7-22, 2013. ISSN 2317-0840.

NORMANDO, D.; ALMEIDA, M. A. D. O.; QUINTÃO, C. C. A. Análise do emprego do cálculo amostral e do erro do método em pesquisas científicas publicadas na literatura ortodôntica nacional e internacional. Dental Press Journal of Orthodontics, v. 16, n. 6, p. 33-35, 2011. ISSN 2176-9451.

MATTIOLI, R. Á. et al. Associação entre força de preensão manual e atividade física em idosos hipertensos. Revista Brasileira de Geriatria e Gerontologia, v. 18, n. 4, p. 881-891, 2015. ISSN 1809-9823.

FERNANDES, A. D. A.; MARINS, J. C. B. Teste de força de preensão manual: análise metodológica e dados normativos em atletas. Fisioterapia em movimento, v. 24, n. 3, p. 567-578, 2011. ISSN 0103-5150.

PÍCOLI, T. D. S.; FIGUEIREDO, L. L. D.; PATRIZZI, L. J. Sarcopenia e envelhecimento. Fisioterapia em Movimento, v. 24, n. 3, p. 455-462, 2011. ISSN 0103-5150.

LENARDT, M. H. et al. Fragilidade e qualidade de vida de idosos usuários da atenção básica de saúde. Revista Brasileira de Enfermagem, v. 69, n. 3, p. 478-483, 2016. ISSN 0034-7167.

BAUMGARTNER, R. N. et al. Body composition in elderly people: effect of criterion estimates on predictive equations. The American journal of clinical nutrition, v. 53, n. 6, p. 1345-1353, 1991. ISSN 0002-9165.

BOBOS, P. et al. Measurement Properties of the Hand Grip Strength Assessment: A Systematic Review With Meta-analysis. Archives of Physical Medicine and Rehabilitation, v. 101, n. 3, p. 553-565, 2020/03/01/ 2020. ISSN 0003-9993. Disponível em: http://www.sciencedirect.com/science/article/pii/S0003999319313668 >.

CANDELORO, J. M.; CAROMANO, F. A. Efeito de um programa de hidroterapia na flexibilidade e na força muscular de idosas. Revista brasileira de fisioterapia, v. 11, n. 4, p. 303-309, 2007. ISSN 1413-3555.

VALE, R. G. D. S. et al. Efeitos do treinamento resistido na força máxima, na flexibilidade e na autonomia funcional de mulheres idosas. Rev. bras. cineantropom. desempenho hum, 2006. 
REBELATTO, J. R. et al. Influência de um programa de atividade física de longa duração sobre a força muscular manual e a flexibilidade corporal de mulheres idosas. Brazilian Journal of Physical Therapy, v. 10, n. 1, p. 127-132, 2006. ISSN 14133555 .

MORI, H.; TOKUDA, Y. Differences and overlap between sarcopenia and physical frailty in older community-dwelling Japanese. Asia Pacific journal of clinical nutrition, v. 28, n. 1, p. 157, 2019. 